\title{
Improving surface quality in selective laser melting based tool making
}

\author{
Filippo Simoni $^{1} \mathbb{D} \cdot$ Andrea Huxol $^{1} \cdot$ Franz-Josef Villmer $^{1}$
}

Received: 22 April 2020 / Accepted: 18 December 2020 / Published online: 2 April 2021

(c) The Author(s) 2021

\begin{abstract}
In the last years, Additive Manufacturing, thanks to its capability of continuous improvements in performance and costefficiency, was able to partly replace and redefine well-established manufacturing processes. This research is based on the idea to achieve great cost and operational benefits especially in the field of tool making for injection molding by combining traditional and additive manufacturing in one process chain. Special attention is given to the surface quality in terms of surface roughness and its optimization directly in the Selective Laser Melting process. This article presents the possibility for a remelting process of the SLM parts as a way to optimize the surfaces of the produced parts. The influence of laser remelting on the surface roughness of the parts is analyzed while varying machine parameters like laser power and scan settings. Laser remelting with optimized parameter settings considerably improves the surface quality of SLM parts and is a great starting point for further post-processing techniques, which require a low initial value of surface roughness.
\end{abstract}

Keywords Direct rapid tooling · Toolmaking - Additive manufacturing process chain · Process control · Production systems $\cdot$ Selective laser melting $\cdot$ Surface roughness $\cdot$ Laser surface remelting

\section{Introduction}

To meet the daily rising global requirements such as efficiency, customization, and improved quality, new and innovative products are being developed continuously with significantly enhanced performance (Zwan 2012). Such novel products should be produced with even more sustainable manufacturing technologies, capable of delivering higher productivity rates, reduced energy and material consumption, and thus with lower environmental impacts (European Commission 2016). For this reason, various manufacturing processes such as milling, laser machining, additive manufacturing (AM) and metal injection molding have been employed in fabricating new metallic products as they can

Filippo Simoni

filippo.simonits@gmail.com

Andrea Huxol

andrea@huxol.eu

Franz-Josef Villmer

franz-josef.villmer@th-owl.de

1 Department of Production Engineering and Management, OWL University of Applied Sciences and Arts, Lemgo, Germany provide reliable solutions and greater geometrical complexity (Chu et al. 2014).

However, independent manufacturing processes often fail to deliver products that meet all the requirements concerning geometrical accuracy, complexity and overall manufacturing costs (Chu et al. 2014; Lauwers et al. 2014). This is due to their natural technological limitations such as their capacity for processing certain materials only, excessive production costs or their inability to produce complex geometries (Newman et al. 2015).

\section{Integration of traditional and additive manufacturing processes}

To address the limitations of autonomous processes, the research community has proposed more innovative and flexible production solutions that integrate two or more technologies. These novel ways of manufacturing are also known as "hybrid manufacturing systems", and are achieved by merging the capabilities of individual technologies and thus benefit from their capacities of being complemental (Chu et al. 2014; Newman et al. 2015; Flynn et al. 2016). For example, the combination of laser-based additive manufacturing technologies with subtractive machining processes is 
currently one of the most important research and development directions in hybrid manufacturing (Fig. 1).

This is primarily due to these processes' capabilities of adding and removing material selectively with controlled resolution and thereby addressing geometrical complexity in parts (internal and overhanging features) while reducing the material wastage and excessive cutting tool usage simultaneously (Flynn et al. 2016; Karunakaran et al. 2010).

In opposite to hybrid machines, current studies with the intent to resolve the limitations of single setup systems, are focused on multi-setup manufacturing solutions, also referred to process chains. With these solutions, the capabilities of complementary manufacturing processes are combined using their sequential integration into multiple machine setups' production lines. Another important advantage of the process chain approach in combining manufacturing technologies is that it can deliver much higher productivity rates due to the parallel utilization of integrated operations and greater overall flexibility (Penchev et al. 2014).

\section{Benefits in tool making}

The direct generation of tools, or direct rapid tooling, is an important field of application for additive manufacturing. There have been several examples of benefits of AM creating lighter structures, combining several components into one, as well as the possibility to customize products for personal or industrial use (Baturynska and Martinsen 2020). Among all the available technologies, Laser Powder Bed Fusion of metals (M-LPBF) plays an important role in producing metal prototypes and is increasingly used for the production of end-use products. The parts produced with Selective Laser Melting (SLM) show comparable mechanical properties to those of forged or cast metal parts (Fousová et al. 2015).

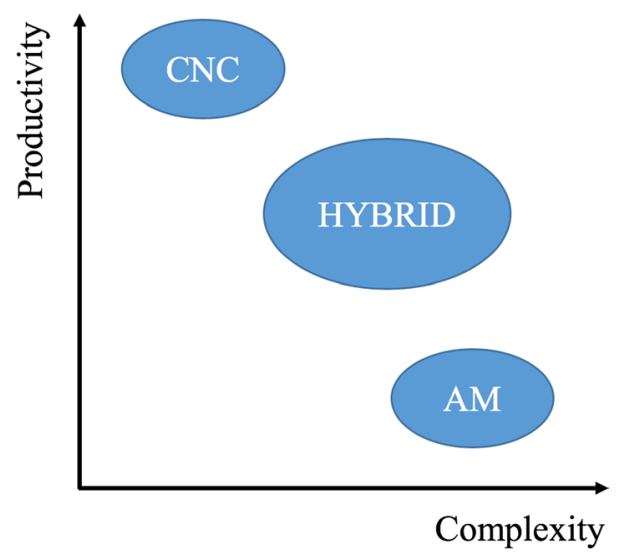

Fig. 1 Comparison between pure additive and subtractive processes and hybrid process
Nowadays one of the key challenges to make this technology robust and cost-effective is to ensure that the designed tools are additively manufacturable. This can be decomposed into two properties: printability and fragility. The printability of an object is the ability of a given additive manufacturing process to realize the desired object. The fragility of the manufactured part is the charachteristic of the object to tolerate successive post-processing stages (Mycroft et al. 2020).

However, there are also other limitation in all current SLM applications: the lack of smooth and even surfaces of the built part. Depending on the geometrical shape and the used machine parameters, the surface can appear rough and partially wavy. This characteristic inhibits the application of SLM built molds for the injection molding process directly after the build process. Usually, the printed molds are postprocessed by the use of subtractive CNC machining or EDM. On the one hand, these post-processing techniques allow the demanded surface qualities and target dimensions. On the other hand, the expected time and resource savings of AM are wasted or even over-compensated.

Surface roughness and unevenness are fundamental realities of the SLM build process. These challenges must be mitigated and solved to avoid many of the problems they may cause.

In the traditional manufacturing industry, the production of prototyping molds can be very expensive and time-consuming and involves high economical risk. Additional costs can come up if the molds need to be changed or modified, and huge losses might be possible if the entire product concept is rejected.

The target is to apply SLM cost-effectively and to integrate it into the process value chain, as presented in Fig. 2.

Therefore, some fundamental improvements are necessary. Among them is the need to optimize the quality of the SLM final products in terms of surface finishing. This is the first step in reducing the need for further post-processing and longer process chains (Elstermeyer and Villmer 2017).

\section{Process chain stages}

The entire process chain consists of several phases. These are described with particular attention to the phases related to the additive manufacturing processes.

\section{Product design}

Nowadays, the majority of the manufacturing processes starts from a software model that completely describes the geometry of the part being produced. Almost any professional CAD solid modeling software can be used, and the output must be a complete 3D solid or surface representation (Gibson et al. 2010). 


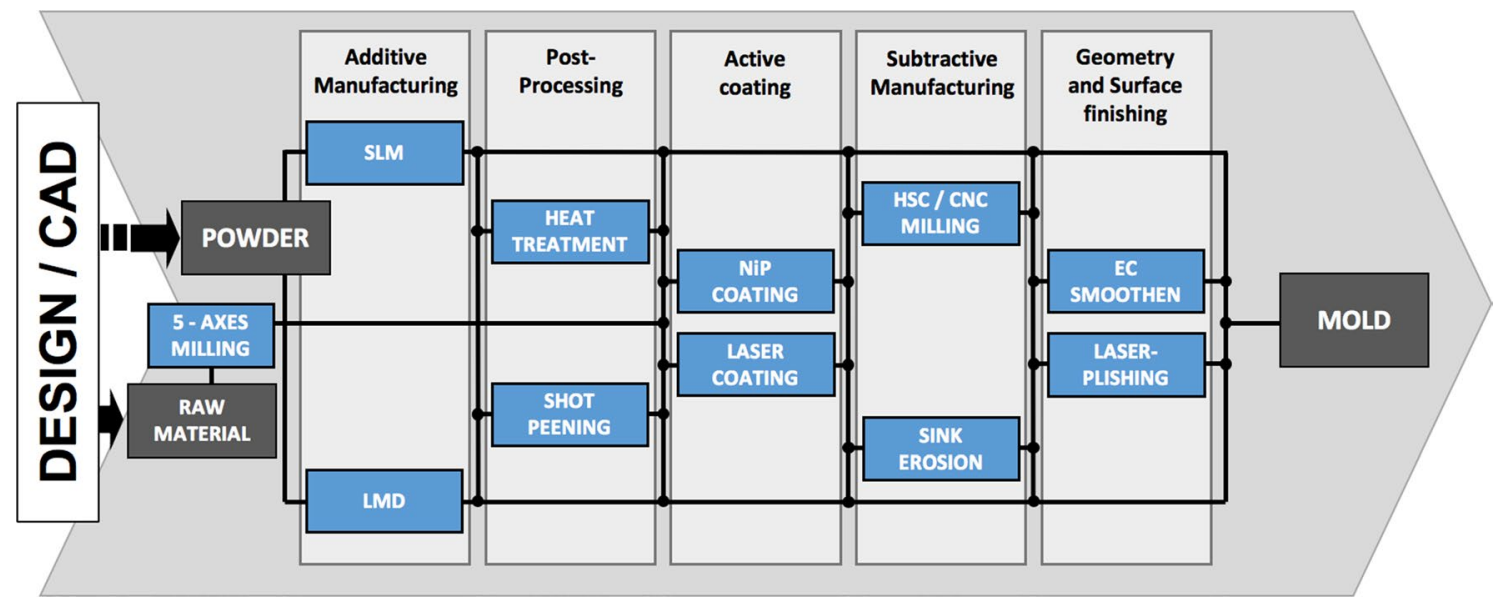

Fig. 2 Proposed process chain

\section{Additive manufacturing processes}

Between all the technologies available in the field of AM, Selective Laser Melting and Laser Metal Deposition (LMD) are the most promising technologies for the production of tools for injection molding. Their layer-by-layer building strategy allows high design freedom and enables building geometrically complex parts. Weight reduction, material recycling and a shorter time to market than traditional manufacturing technologies are further important advantages. For this use case, SLM offers the opportunity to print, for instance, complex conformal cooling channels leading to reduced cycle times. The applicability and effectiveness strongly depend on the shape of the mold. The more difficult it is to dissipate heat from the tool, the more effective is the integration of conformal cooling channels. SLM is not limited to the accessibility of tools, thus almost every shape can be printed. At the end of the build process, the remaining powder can be sieved and mostly reused, which is again a strong economic and resource-specific advantage (Elstermeyer and Villmer 2017).

A typical characteristic of SLM is the required connection between the part and the base substrate and necessary support for overhangs with more than $40^{\circ}$. These mechanical supports are needed to prevent thermal deformations of the built part. The removal of the support structures is highly time-consuming and is most often carried out manually. This is also one of the main causes of poor surface quality. Thus, in mold making, it is recommendable to avoid support structures attached to areas relevant to the demolding of plastic parts. In general, printed surfaces appear rough with roughness values far higher than required for a proper removability of plastic parts from the mold. A typical average roughness value of $\mathrm{Ra}=12 \mu \mathrm{m}$ is achievable with SLM technology (Yasa and Kruth 2011). Another aspect is, that a proper ejection of solidified polycarbonate, requires draft angles of $6^{\circ}-7^{\circ}$ (Menges 1991). This drastically limits the freedom of design of the end product and over-compensates the advantages of direct rapid tooling. The roughness of the print surfaces increases with decreasing the draft angle and reaches a maximum for horizontal top layers. Due to a low powder bulk density, the powder shrinks unevenly. This leads to mountain-like top textures (Unterberg 2016).

Analytical and experimental tests prove, that the staircase effect of layer-wise build-up increases the tension and shear stresses of AM parts (Scheideler et al. 2016). Therefore, it can be expected, that the imprints of such textures into the injected plastic will consequently reduce the loading capacity of molded plastic, too.

\section{Subtractive manufacturing}

High-Speed Cutting and Computer Numerical Control (CNC) machining are the best performing subtractive manufacturing processes in terms of surface finishing and geometrical precision. These technologies will fail only in the case of very deep, sharp or highly twisted features due to the lack of accessibility and non-availability of specific cutters. Even they can be addressed with subsequent processes such as EDM.

Advances in machine and cutting tools have made highspeed machining a reality. Despite the continuous improvement, the current bottleneck of CNC machining is the CAM software. Existing CAM packages, which are essentially 3D geometric modelers, can generate almost optimal and collision-free cutter paths, but most of the time, they do not guarantee safe or efficient cutting parameters. The machining parameters are often specified only once at the beginning of the process, and may not be optimal throughout the procedure due to varying axial and radials depths of cut. Adaptive 
control can overcome this difficulty through in-process force measurements but it lacks reliability and it is very expensive (Karunakaran et al. 2012).

\section{Post-processing}

Usually, heat treatments have been found to improve ductility but at the same time also degrade strength by forming coarse grain particles. In different practical applications, mechanical and surface properties such as hardness and surface roughness play a significant role (especially in devices with involved moving parts) in determining the life and performance of the components. This implies that, for some specific applications, heat treatment might not be a suitable post-process to enhance SLM-fabricated high-performance parts.

Generally, aging heat treatments cannot eliminate internal defects such as pores and micro-cracks. To reduce the porosity, a Hot Isostatic Pressing (HIP) process, which combines high pressure and high temperature, may be applied. The influence of the HIP process parameters on the density and microstructure of IN718 SLM components is well studied and analyzed by (Tillmann et al. 2017). Their results showed that the majority of pores were densified employing HIP (Bodziak et al. 2019).

\section{Geometry and surface finishing}

Amongst the available processes to enhance the requested geometrical tolerances and surface finishing, different plasma electrolytic processes have gained attention due to their capability to enhance surface properties (Parfenov et al. 2015). Depending on the processing parameters and conditions, electrolytic plasma processes can be divided into oxidation, coating, surface cleaning, and polishing. In the Plasma electrolytic Polishing (PeP) plasma electrolysis takes place in addition to the classic electrolysis. $\mathrm{PeP}$ is primarily determined by the dissolution of the anode and plasmachemical reactions. With an optimized process setup, it can be utilized to finish machined surfaces of complex metallic parts. Components of almost any shape can be processed as no shaped electrode is applied in the PeP process. The most important feature of this promising technology is that environmentally friendly aqueous electrolytes are used, instead of the acids, harsh and toxic electrolytes, as used for other electrochemical polishing techniques. Achievable roughness values of $\mathrm{Ra}$ around $0.02 \mu \mathrm{m}$ are possible and manageable removal rates make $\mathrm{PeP}$ an interesting technology for precision parts and functional surface optimization. The achievable surface properties by PeP depend on the initial state of the workpiece surface, but in general, there is low or no necessity for pre-treatment (surface impurities or residues are well removed during the PeP process) (Nestler et al. 2016).

\section{Active coating}

For precise surface modifications or high-quality coatings, ceramic materials find extensive applications thanks to their exceptional hardness, excellent wear resistance, and stability at high temperatures (Wang et al. 2004). High power lasers are emerging as efficient tools for the deposition of wear-resistant coatings. Laser coating is a fast and reliable process applicable to many engineering components without the requirement of elaborate support systems (e.g. ultrahigh vacuum environment, etc.). In this technology, high cooling rates allow the formation of extremely fine-grained structures and improved mechanical properties (Agarwal and Dahotre 1999). In most cases, laser treatment also ensures strong metallurgical bonding between the coating and substrate, resulting in a well-optimized surface quality (Masanta et al. 2009).

\section{Surface quality of SLM parts}

Nowadays with metal AM technologies, it is not possible to achieve surface qualities as good as generated via NC machining. Different aspects have to be taken into account to ensure the capability of additively manufactured mold to be used in production. Among other parameters, such as surface form, surface waviness and surface hardness, a very important figure is the surface roughness.

The surface roughness of the SLM parts is highly dependent on many factors and process parameters. Among them, the grain size of powder particle, layer thickness, wall angle, and melt pool size are the most relevant (Fraunhofer 2015). The wall angle parameter is defined as the angle between a specific surface and the horizontal plane. Figure 3 shows the influence of the wall angle and layer thickness on the surface roughness.

The combination of wall angle and layer thickness produces the well-known staircase effect. The stair size decreases proportionally with the cosine of the wall angle. Consequently, the staircase effect can be reduced by decreasing the layer thickness or by increasing the wall angle.

In both cases, more stairs appear, but the size of the stairs becomes smaller, leading to lower surface roughness. For very high-wall angles $\left(>75^{\circ}\right)$ roughness is not improving any further, because the staircase effect does not play a role anymore and other effects as balling, cause the roughness to increase slightly. The top surface roughness (at $0^{\circ}$ ) improves strongly for smaller layer thicknesses because higher thermal conductivity and the presence of less powder lead to smaller and more stable melt pools. The effect of layer thickness on 
Fig. 3 Influence of wall angle on surface roughness (Yasa 2011)

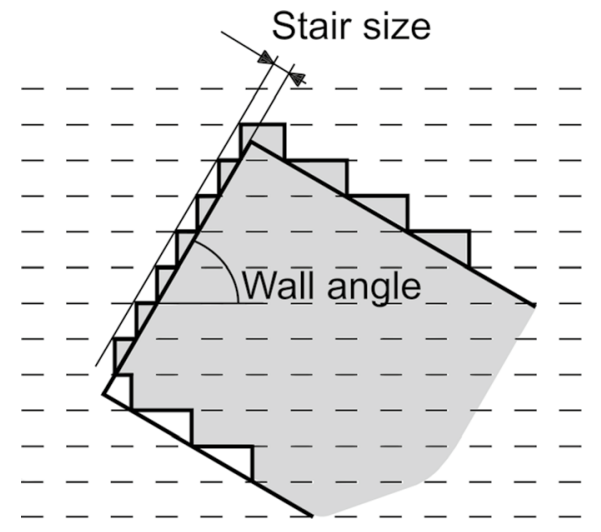

side surface roughness (at $90^{\circ}$ ) is less prominent because a good overlap between successive layers is still reached for a layer thickness of $50 \mu \mathrm{m}$.

Down-skin or overhanging surfaces with a build angle below $60^{\circ}$ have a high roughness. Moreover, bottom surfaces with a sloping angle below $40^{\circ}$ are not achievable without support structures. Bottom surfaces are not finished well because of two reasons. Firstly, since the laser beam scans on loose powder instead of on solid material, the thermal conductivity decreases and the temperatures increase leading to unstable melt pools. Secondly, stalactite patterns are formed because the molten material sinks into the loose powder by gravity. When overhanging surfaces with lowsloping angles cannot be avoided by tilting the part, specific laser parameters for the first few layers above the overhanging surface or support structures should be used (Vandenbroucke and Kruth 2007).

The work of Elstermeyer and Villmer (2017) validates these outcomes and proves that worst results for up-skin surfaces are obtained for the top flat surfaces (surfaces with a normal vector parallel to the building axis).

Optimizing the process parameters can limit this undesired effect, but not completely avoid it. Hence, some other solutions are needed that can help to overcome the low obtainable surface quality for up-skin layers.

\section{Optimal tool design}

Surface roughness is the most relevant factor for the ability to demold plastic parts from a molding post. The lowest roughness values can be achieved at vertical walls with $3^{\circ}-20^{\circ}$ of positive inclination (Fig. 4). Therefore, the advisable draft angle for a minimum of post-processing is found at around $3^{\circ}$, which is equivalent to draft angles in standard tools. Subsequently, it can be concluded that: for the alignment of the mold within the build envelope, it is useful to deploy the substrate plate as a fixation to the stem tool. Thus, the print can easily be assembled to a stem tool. As a consequence, the print has to be aligned with the aperture

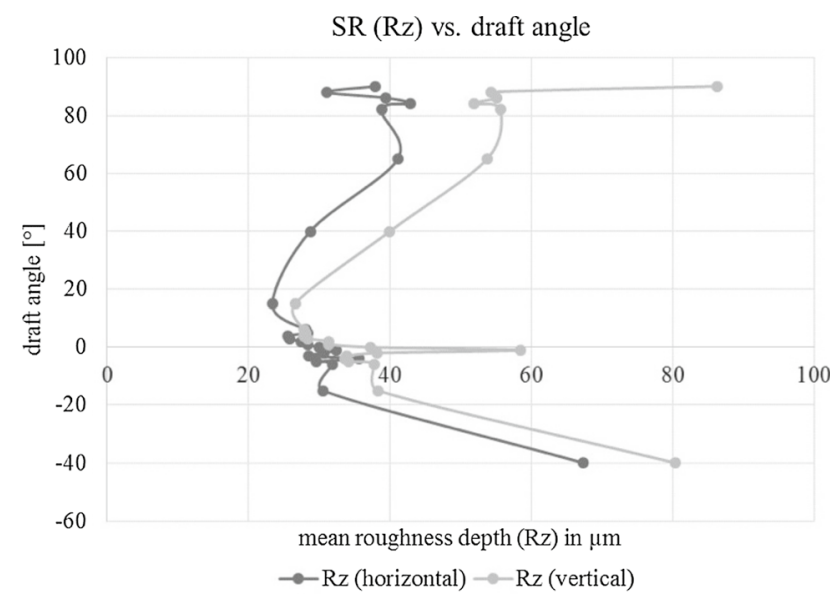

Fig. 4 Impact of wall angle (draft angle) on surface roughness (Elstermeyer and Villmer 2017)

to the top, which further brings the lowest roughness to vertical walls and reduces the necessity of post-processing significantly.

Cooling channels should not have any functional crosssections. To avoid support structures, cooling channels can either be printed with oval or diamond shapes. Also, they should be orientated horizontally in the build process. However, the profile for vertically aligned channels is freely selectable (Verein Deutscher Ingenieure e.V. 2013). Besides, the top horizontal layers should not be undergone with channels at less than $5 \mathrm{~mm}$ distance. This avoids aftereffects from powder sacking in levels where the channels are generated. A material offset needs to be designed to enable accurate drilling, ribbing or erosion after print. For mechanical postprocesses like glass blasting, grinding and polishing operations, it is recommended to split the tool design so that inaccessible gaps can easily be reached (Elstermeyer 2017).

For the application in Rapid tooling, a lower roughness is highly needed, especially for functional and visual sidewalls. For functional walls, as e.g. walls provided with draft angles, it is important to achieve a smooth surface to ensure 
Table 1 Draft angles at different surface finishes (according to Newnes 2015)

\begin{tabular}{lccccc}
\hline Charm. $-\mathrm{Nr}$ & $\mathrm{Ra}$ & $\sim \mathrm{Rz}$ & \multicolumn{3}{l}{ Draft angle $\mathrm{x}^{\circ}$} \\
\cline { 5 - 6 } & $(\mu \mathrm{m})$ & $(\mu \mathrm{m})$ & $\mathrm{PA}$ & $\mathrm{PC}$ & $\mathrm{ABS}$ \\
\hline 12 & 0.40 & 1.5 & 0.5 & 1.0 & 0.5 \\
15 & 0.56 & 2.4 & 0.5 & 1.0 & 0.5 \\
18 & 0.80 & 3.3 & 0.5 & 1.0 & 0.5 \\
21 & 1.12 & 4.7 & 0.5 & 1.0 & 0.5 \\
24 & 1.60 & 6.5 & 0.5 & 1.5 & 1.0 \\
27 & 2.24 & 10.5 & 1.0 & 2.0 & 1.5 \\
30 & 3.15 & 12.5 & 1.5 & 2.0 & 2.0 \\
33 & 4.50 & 17.5 & 2.0 & 3.0 & 2.5 \\
36 & 6.30 & 24.0 & 2.5 & 4.0 & 3.0 \\
39 & 9.00 & 34.0 & 3.0 & 5.0 & 4.0 \\
42 & 12.50 & 48.0 & 4.0 & 6.0 & 5.0 \\
45 & 18.00 & 69.0 & 5.0 & 7.0 & 6.0 \\
\hline
\end{tabular}

the removability of the plastic parts after solidification. If the surface roughness tends to be too high, the plasticized polymer sticks onto the mold walls and cannot be removed from the cast, causes scratches or damages to the part. The ability to deform the injected plastic is heavily dependent on the surface roughness, corresponding to the draft angle. According to Table 1, it is principally possible to demold plastic parts even with relatively high roughness in the side walls.

Thus, the higher the achieved roughness, the higher the inclination of draft angles is required. The need for increased draft angles then again influences the shape of the injectionmolded target product. This could lead to a dysfunction of the part and enormous design changes. The requirement of transparent faces, furthermore, cannot be fulfilled by a rough surface. Such faces generally require a polished finish which SLM cannot approach to.

\section{Measure the surface roughness}

As given in Table 1, two values are meaningful enough to predict the ability of the injected plastic to demold from the tool. First is Ra, which "is calculated by the arithmetic average of the absolute values of the profile height deviations from the mean line, recorded within the evaluation length" (Harrison Electropolishing L.P. 2016). Further importance is given to Rz, the mean roughness depth. The value is related to the tendency of plastic to stick on the sidewalls of the mold. The higher the amplitude of the roughness swings, the more difficult it is to remove the plastic after cool down. This attachment can cause scratches in the plastic or destroy the part entirely.

To measure the surface roughness, one of the most used methods is the tactile analysis. A stylus instrument enables the measurement of roughness via a keying head holding a needle which is automatically moved over the surface (Fig. 5).

According to the norms DIN EN ISO 4288:1998 and DIN EN ISO 3274:1998, the track distance the keying head moves over the surface, also known as traversed length $(\mathrm{Lt})$, depends on the expected surface roughness. For SLM built parts a roughness of $2.00<\mathrm{Ra}<10.0 \mu \mathrm{m}$ and $10<\mathrm{Rz}<50 \mu \mathrm{m}$ is expected, which leads to a default Lt of $12.5 \mathrm{~mm}$. For the testing of parts produced by SLM, this means that the default traversed length influences the design of all test objects (Mitutoyo Europe $\mathrm{GmbH}$ ).

The influence of different materials on draft angles represents another important aspect that can be analyzed from Table 1. Polycarbonate is hereby representing the most challenging material to be deformed. Due to the draft angle limited to $3^{\circ}$ inclination, as given in the specification book, the targeted Roughness values for polycarbonate are Ra: $4.5 \mu \mathrm{m}$, Rz: $17.5 \mu \mathrm{m}$.

As already mentioned, the final surface roughness obtainable with the majority of the AM processes is not as good as with standard manufacturing techniques. This is mainly due to the nature of the process and, for powder bed processes, to the behavior of the powder material. Partially molten powder particles, not optimal machine parameters as well as the needed support structures for inclined surfaces drastically reduce the surface smoothness of the processed parts. This issue could be solved during three steps of the global process chain: in the Pre-process, In-process or Post-process phases.

\section{Optimization stages}

The Pre-process phase represents the initial design of the part (product design phase). Once the machining technology is chosen and with it its characteristics, for obtaining the required final properties it is necessary to develop and arrange the design of the part according to the process capability. This means, regarding the metal powder bed AM processes as SLM, that a studied and smart design of the part and its orientation inside the build chamber of the machine

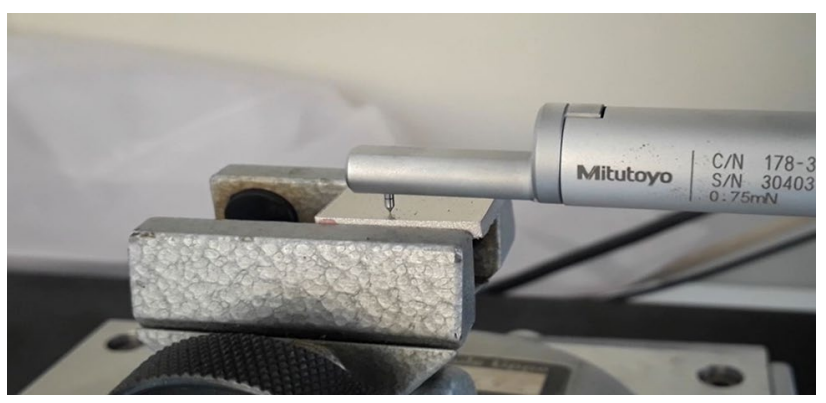

Fig. 5 Surface roughness measurement via a stylus instrument 
is required, e.g. to reduce the need for support structures and issues related to the orientation of the surfaces with the building direction. In-process optimization, as the name indicates, intends to optimize the process and its parameters to allow the production of the part with the desired behaviors without or with a reduced need for post-processing. In this direction, the work of Ning et al. (2019) investigates the correlation between the in-process temperature and residual stresses and distorsion of parts produced with metal powder bed additive manufacturing (MPBAM). The last step, the post-process phase, is nowadays required by all the AM technologies, as they do not permit a direct utilization of the built parts without a minimum of post-treatment (as active coatings, heat treatments or improvement in surface qualities).

Amongst all the techniques used to improve the final surface quality and to reduce the process chain complexity, the In-process approach is the most interesting, as it grants very good results at the cost of slightly longer production time (Yasa et al. 2011). By the use of in-situ melt pool temperature monitoring, it is also possible to predict the powder packing porosity and thus the behavior in terms of mechanical and physical properties of the final part (Ning et al. 2019).

As the SLM process plays an important role in the toolmaking industry, an optimization of the process towards the final obtainable part quality is nowadays in high demand. While SLM provides many benefits compared to conventional machining, low surface quality is one of its major limitations (Yasa et al. 2011). As already mentioned, the surface roughness of the parts is highly dependent on the build angle between the surfaces and the horizontal plane, and the worst results are obtained for top flat surfaces (surfaces with a normal vector parallel to the building axis).

Laser remelting is a promising feature for powder bed processes. Many benefits can be obtained with this technique, from a higher part density and better microstructure to reduced surface roughness. After the complete processing of a single layer, the same slice is scanned again. This practice slightly increases the production time but at the same time, it can be the best solution for applications where residual porosity or poor surface finishing are undesired (Yasa et al. 2011).

\section{Laser surface remelting}

For top flat surfaces, laser remelting (LSR) may be an easy solution without removing the part from the building platform and avoiding fixation errors. LSR is capable of producing a high-performance top layer with strong metallurgical bonding to the substrate. This is obtained with several rapid remelting and solidification conditions (as schematically represented in Fig. 6). This process ensures significant grain

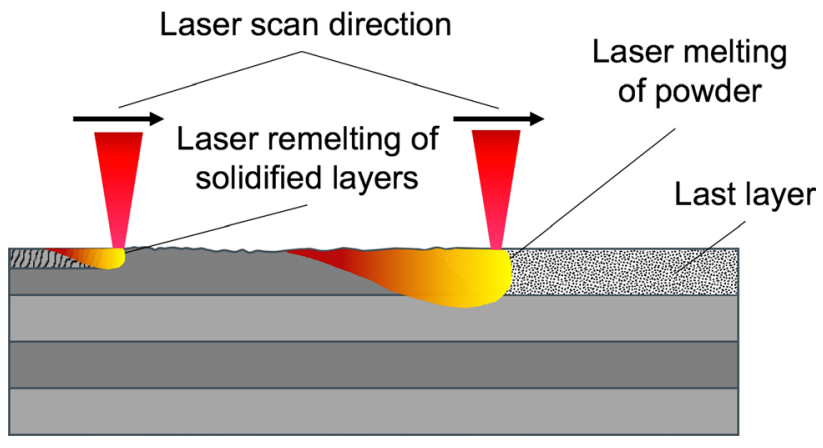

Fig. 6 Schematic representation of the working principle of laser surface remelting

refinement and uniformity of the microstructure on the surface (Zhong et al. 2006).

To investigate the potential to decrease the surface roughness values of horizontal surfaces, the LSR process is applied only after the part is completely made by SLM and the last slice info is used for laser remelting.

This technique to improve the surface finishing of the SLM part can be easily applied to top flat surfaces. However, it can be possible to take advantage of this method also to improve the surface quality of inclined walls. In this case, other phenomena are causing higher roughness such as the staircase effect and balling (that are due to the layer-wise building of the parts and the powder nature of the material). In this case some other machine parameters have to be optimized.

\section{Experimental setup}

To quantify how LSR can be efficient in the surface optimization of SLM parts, an experimental setup is designed and four build jobs are performed. The machine used is a Realizer SLM 125 and the material a CoCr alloy with a particle size of $10-55 \mu \mathrm{m}$. This material is largely used in biomedical applications, mainly for dental and orthopedic implants and prosthesis. The designed samples have dimensions of $15 \times 20 \times 3 \mathrm{~mm}^{3}$. In the first three build jobs, a total number of 36 samples with $90^{\circ}$ build angle are produced (12 samples in each build job), to analyze the influence of different LSR parameters on up-skin layers (Fig. 7).

In the fourth build job, designed to apply LSR to inclined surfaces, the sample are built as it follows: six samples with $0^{\circ}$ build angle, six samples with different build angles $\left(15^{\circ}\right.$, $30^{\circ}, 45^{\circ}, 60^{\circ}, 75^{\circ}$, and $90^{\circ}$ ) not subject to remelting and six samples with the same build angles but with a remelting of the contour scans (Fig. 8).

The sets of remelting parameters changed from sample to sample leading to a large number of results to investigate 
Fig. 7 Design of the first three build jobs

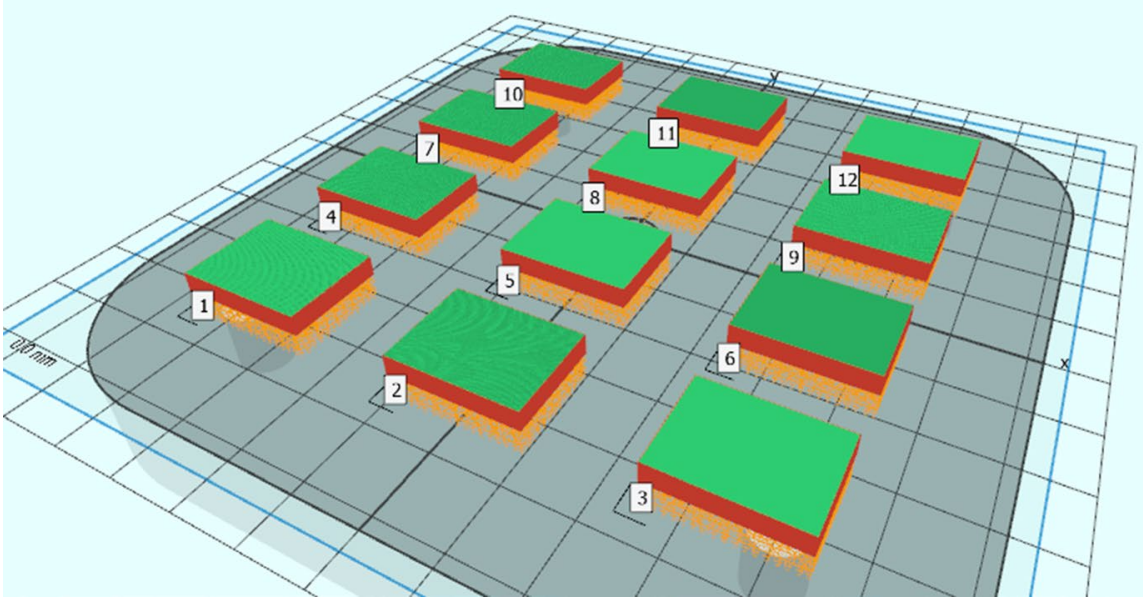

Fig. 8 Design of the fourth build job

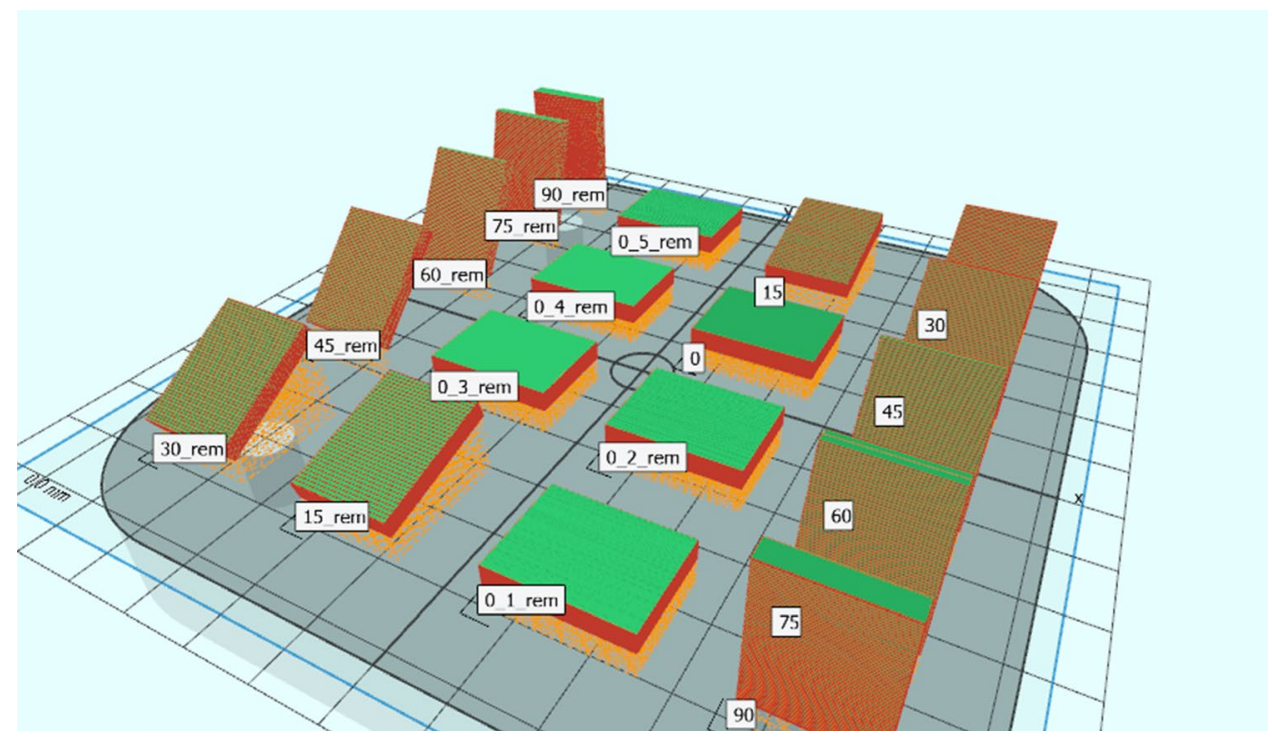

the correlation between machine parameters and outcomes. This is possible by varying some important process parameters. Using the standard machine parameters for the $\mathrm{CoCr}$ powder, different combinations of laser power, scan speed, and beam spot diameter values, as well as the different number and orientation of the remelting exposures are tested (Table 2).

In the first two build jobs, four samples are produced with standard machine parameters and without remelting, to be able to compare the results with the parts exposed to LSR.

In the first three build jobs, the number and orientation of the remelting exposures are also taken into consideration.

After the parts are built and unpacked, their surfaces are tested with a stylus surface roughness measuring machine, a Mitutoyo SURFTEST SV-3200. The data is
Table 2 Process parameter variation

\begin{tabular}{lll}
\hline Process parameter & Unit & Values range \\
\hline Laser power & $\mathrm{W}$ & $88 \div 106$ \\
Scan speed & $\mathrm{mm} / \mathrm{s}$ & $250 \div 1000$ \\
Beam spot diameter & $\mu \mathrm{m}$ & $37 \div 100$ \\
Number of remelting & - & $0,1,3$ \\
Angle btw. remelting tracks & $\left({ }^{\circ}\right.$ & $0,45,90$ \\
\hline
\end{tabular}

then analyzed with the proprietary software and the surface roughness profiles are exported. For each sample, the average values of $\mathrm{Ra}$ and $\mathrm{Rz}$ calculated after three measurements are taken into consideration. 


\section{Experimental results and discussion}

\section{Up-skin layers}

The first three tests are built to search which set of machine parameters lowers the surface roughness for samples with a $0^{\circ}$ build angle the most. Every sample is built with different combinations of remelting parameters. All the layers are scanned with parameters that are optimized for density. The last layer of each sample is then exposed to laser remelting with scan vectors with an angle of $0^{\circ}, 45^{\circ}$ or $90^{\circ}$ compared to the last scan vector of the melting. Each part is re-scanned (re-molten) with a different combination of scan speed, laser power, and beam spot diameter. The following scan speeds are used: $250 \mathrm{~mm} / \mathrm{s}, 333 \mathrm{~mm} / \mathrm{s}, 500 \mathrm{~mm} / \mathrm{s}, 666 \mathrm{~mm} / \mathrm{s}$, $750 \mathrm{~mm} / \mathrm{s}, 1000 \mathrm{~mm} / \mathrm{s}$. Each of these scan speeds is used in combination with the following laser powers: $88 \mathrm{~W}, 96 \mathrm{~W}$, $100 \mathrm{~W}, 105 \mathrm{~W}, 108,112 \mathrm{~W}$. The laser beam spot diameter assumed these values: $37.40 \mu \mathrm{m}, 48.40 \mu \mathrm{m}, 56.80 \mu \mathrm{m}$, $65.40 \mu \mathrm{m}, 74 \mu \mathrm{m}, 82,60 \mu \mathrm{m}$. On each part, three 2D roughness measurements are done in the direction perpendicular to the remelting vectors. Figure 9 gives the Ra values measured with a gaussian filter and a cut-off of $2.5 \mathrm{~mm}$. The red line is the average Ra value for the four samples not subjected to LSR. The setup parameters that ensured the best results in the first build job are used as a basis for the second one, and so on for the third build job. Selective process parameter optimization allows the production of parts with very interesting results.

Figure 10 shows the roughness profile of the top surface of a sample without surface remelting. It is possible to see how the roughness profile is very irregular, with rough peaks and deep valleys. The measured $\mathrm{Ra}$ value for this part is $10.68 \mu \mathrm{m}$. When analyzing the profile of the top surface of

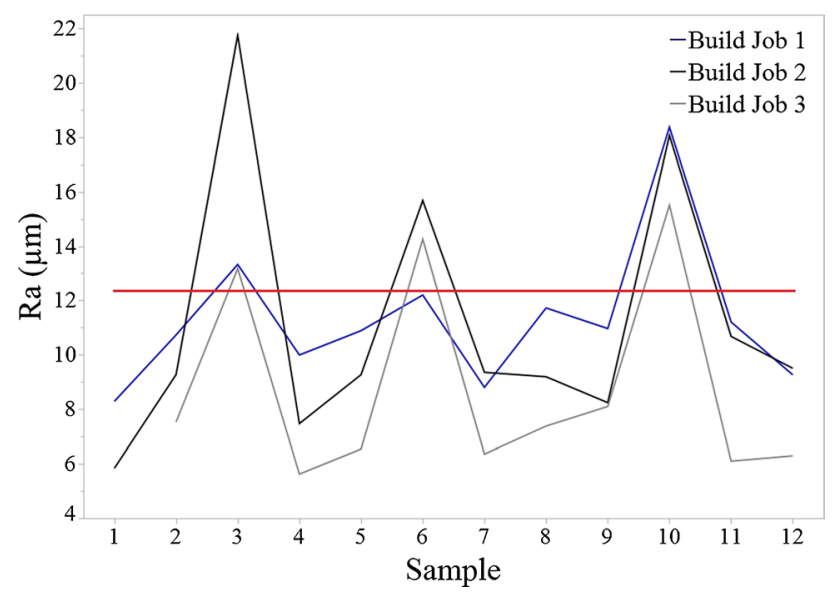

Fig. 9 Surface roughness results of the samples built in the first three build jobs

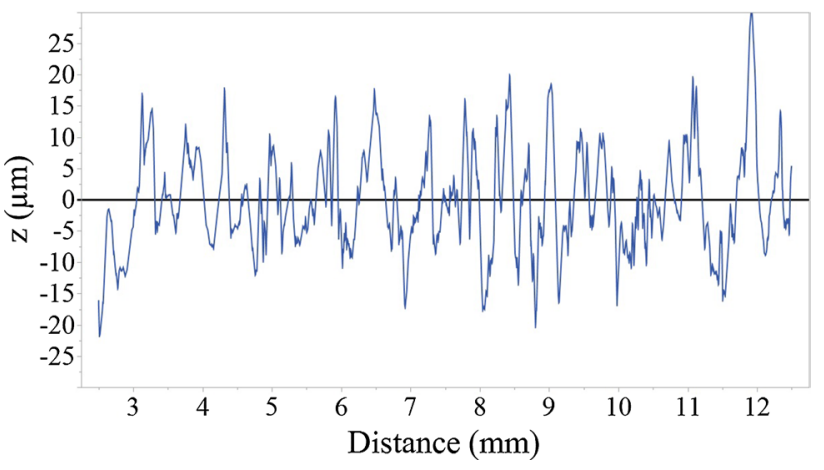

Fig. 10 Roughness profile of an unprocessed SLM part

a sample processed with LSR, it is possible to see how this technique improves the surface quality.

In Fig. 11 the roughness profile of a sample subjected to three remelting cycles is represented. The peaks are smoothened and the valleys are less deep. The measured Ra value for this sample is $5.84 \mu \mathrm{m}$, which corresponds to a reduction of $45 \%$ of the average surface roughness. These plots and the results depict the efficiency of this technique to improve the surface finishing of SLM built parts.

The machine parameters that ensured the best results are a scan speed of $250 \mathrm{~mm} / \mathrm{s}$, a laser power of $105 \mathrm{~W}$, and a beam spot diameter of $65.40 \mu \mathrm{m}$. Of course, these parameters relate to the particular combination of machine and material used for this study.

To characterize the effect of LSR also on the inner section of the samples, a micrographic analysis of an inner cross-section of the samples produced in the build job 2, is conducted. This requires a specific preparation of the samples. After the grinding and polishing, three images of each sample are taken. Using the lower magnification of $50 \mathrm{X}$, it is not possible to capture the upper cross-sections with less than 15 pictures, so it is decided to analyze three images, two on the top corners, Figs. 12 and 13a, c, and one in the middle of the upper part of the samples, Figs. 12 and 13c.

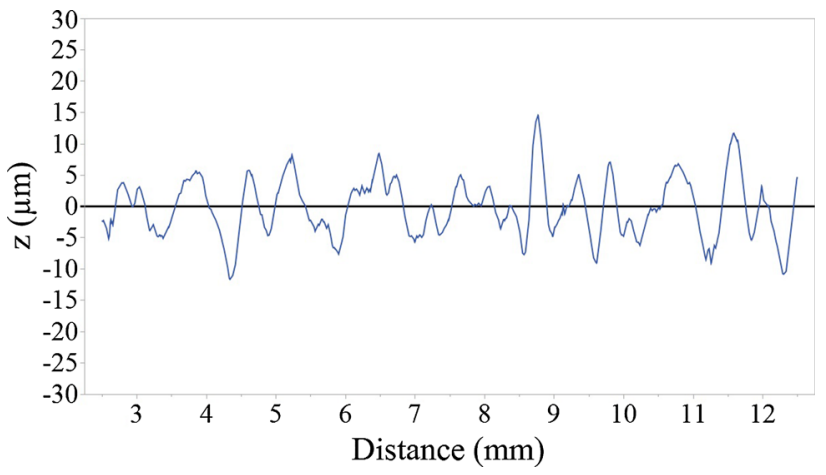

Fig. 11 Surface roughness of an SLM part subjected to three-times remelting 


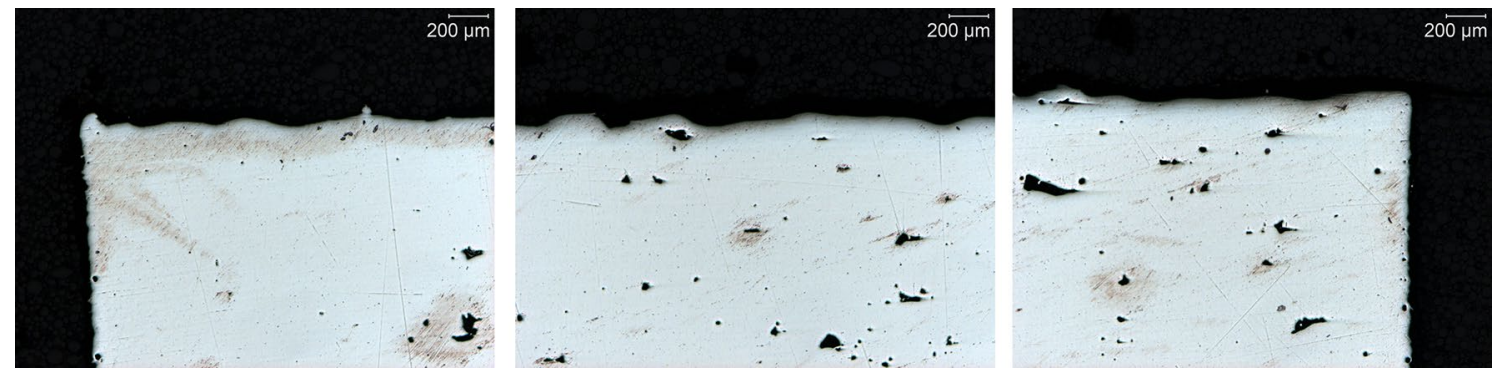

Fig. 12 Cross-section of the top surface of a sample not subjected to LSR
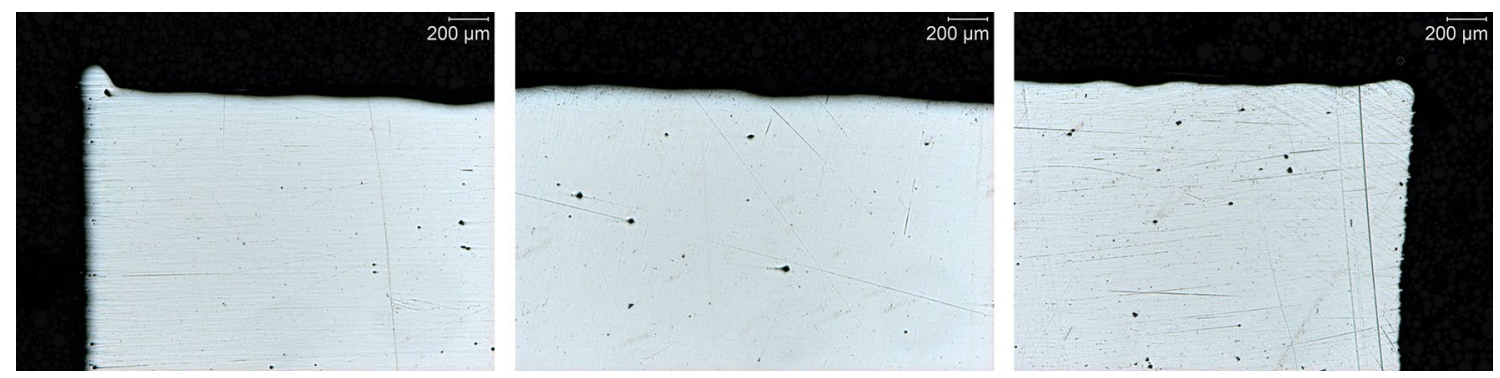

Fig. 13 Cross-section of the top surface of a sample re-exposed three times to LSR

In the following Figs. 12 and 13 the cross-sections of a sample not subjected to LSR and a sample re-exposed three times to LSR are shown.

The differences between the profiles of the two surfaces are highly remarkable. An irregular and rough surface of the unprocessed sample (Fig. 12) is replaced by a smooth and relatively even skin in the re-melted sample (Fig. 13).

Another benefit of this technique that can be noticed in these two pictures, is the reduced porosity just below the surface of the re-melted part. Selective remelting provides the densification of the last layer and thus an overall improvement of the mechanical and physical properties of the upper section. This aspect can be seen in all the samples subjected to LSR.

A negative effect of remelting of the last layer is the height difference that is formed between the inner part of the surface and the contour (as can be seen in Fig. 13a). This is a consequence of the densification of the upper section of the samples that takes place only in the area of the sample that is re-melted. This is because only the hatch is re-exposed to LSR, and not the contour. Different laser parameters are used for these two scanning areas, and the combination of these factors leads to this undesired shape deviation in the top surface of the samples. It has to be evaluated whether this phenomenon could be reduced by re-exposing also the contour of the samples.
Table 3 Roughness results of build job 4

\begin{tabular}{lll}
\hline Sample & $\mathrm{Ra}$ & $\mathrm{Rz}$ \\
\hline 15 & 7.410 & 39.051 \\
30 & 6.979 & 38.722 \\
45 & 5.356 & 32.112 \\
60 & 3.838 & 24.517 \\
75 & 3.468 & 21.782 \\
90 & 3.382 & 19.770 \\
15_rem & 6.120 & 32.928 \\
30_rem & 3.988 & 23.606 \\
45_rem & 3.489 & 20.683 \\
60_rem & 3.415 & 19.694 \\
75_rem & 3.679 & 20.264 \\
90_rem & 2.756 & 16.031 \\
\hline
\end{tabular}

\section{Inclined surfaces}

The surface roughness values for the inclined samples of the fourth build job are summarized in Table 3. It is possible to appreciate, the dependency between build angle and surface roughness. As reported in many other studies (Elstermeyer and Villmer 2017), for higher build angles both the surface roughness parameters $\mathrm{Ra}$ and $\mathrm{Rz}$ decrease. This trend is visible, in the following Fig. 14, as well in the sample not 


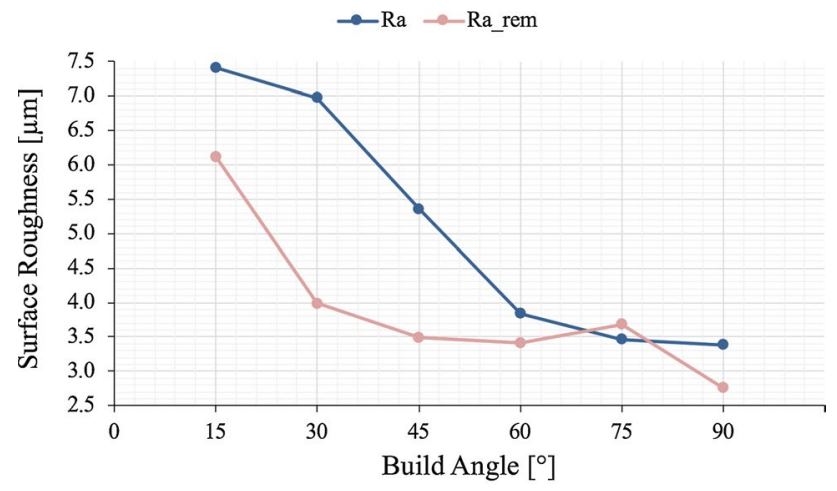

Fig. 14 Dependency between surface roughness and build angle for samples subjected and not subjected to LSR

subjected to LSR (blue markers) as in the sample with a remelting of the contour scan (red markers).

The samples treated with LSR, show a reduced surface roughness up to $43 \%$. In this case, the highest reduction is obtained for the sample with a $30^{\circ}$ build angle. For every sample, a remarkable reduction in surface roughness occurs, except for the sample with a $75^{\circ}$ build angle when the roughness value Ra shows an increase of $6 \%$.

The search for some dependencies between results and process parameters could give a good idea on which combination of machine parameters will result in the best surface quality. In many studies, some important process parameters are integrated within the definition of Energy Density. Laser power, laser scan speed, layer thickness, and hatch distance contribute to the values of energy density. The results show a correlation between energy density and surface roughness. Unfortunately, varying the machine parameters leads us to a wide range of energy density values. Because there are a limited number of roughness results for a lot of energy density values these correlations are not published in this paper. This could be the starting point for future research.

\section{Conclusions and outlook}

With this article, the idea of an integrated process chain with additive and subtractive manufacturing and post-processing techniques is explained and analyzed. Focused attention is paid to the capability of SLM to produce tools for injection molding, taking advantage of its proficiency in building conformal cooling channels and complex geometry. However, the deficit of specially developed tools to avoid integration issues in combining AM with other technologies leads to high confusion and overall risks in producing parts that include geometries with different manufacturing requirements, e.g. parts with areas that can be cost-effectively machined while others require AM solutions. In this context, an optimization towards enhancement in surface roughness of SLM parts is analyzed, and the potentialities of the LSR technique are confirmed, as the results of the performed experiments demonstrate tangible improvement towards better surface finishing in terms of surface roughness. This is also an optimal starting point to integrate SLM with new promising post-processing techniques for the improvement of additively manufactured metal parts, such as Plasma Electrolytic Polishing.

In this paper, some other important induced phenomena of LSR on the processed samples have not been discussed. Induced thermal effect on the molten pool as well as thermal shrinkage after the cool-down and undesired residual stresses, balling effect and keyholing effect play an important role on the overall parts' surface quality. All these influences, combined with the fact that the results are highly dependent on the material and machine used, must drive further research and implementation in the application of LSR to SLM printing technology.

Funding Open Access funding enabled and organized by Projekt DEAL.

Open Access This article is licensed under a Creative Commons Attribution 4.0 International License, which permits use, sharing, adaptation, distribution and reproduction in any medium or format, as long as you give appropriate credit to the original author(s) and the source, provide a link to the Creative Commons licence, and indicate if changes were made. The images or other third party material in this article are included in the article's Creative Commons licence, unless indicated otherwise in a credit line to the material. If material is not included in the article's Creative Commons licence and your intended use is not permitted by statutory regulation or exceeds the permitted use, you will need to obtain permission directly from the copyright holder. To view a copy of this licence, visit http://creativecommons.org/licenses/by/4.0/.

\section{References}

Agarwal, A., \& Dahotre, N. B. (1999). Laser surface engineering of steel for hard refractory ceramic composite coating. International Journal of Refractory Metals \& Hard Materials, 17, 283-293.

Baturynska, I., \& Martinsen, K. (2020). Prediction of geometry deviations in additive manufactured parts: Comparison of linear regression with machine learning algorithms. Journal of Intelligent Manufacturing. https://doi.org/10.1007/s10845-020-01567-0.

Bodziak, S., Al-Rubaie, K. S., Valentina, L. D., et al. (2019). Precipitation in 300 grade maraging steel built by selective laser melting: Aging at $510^{\circ} \mathrm{C}$ for $2 \mathrm{~h}$. Materials Characterization, 151, 73-83.

Chu, W. S., Kim, C. S., Lee, H. T., Choi, J. O., Park, J. I., et al. (2014). Hybrid manufacturing in micro/nano scale: A review. International Journal of Precision Engineering and ManufacturingGreen Technology, 1, 75-92.

European Commission. (2016). Horizon 2020 Work program 20162017: Cross-cutting activities, https://ec.europa.eu/research/parti cipants/data/ref/h2020/wp/2016_2017/main/h2020-wp1617-focus _en.pdf.

Elstermeyer, O., \& Villmer, F.-J. (2017). SLM Based tooling for injection molding-Focus on reduced effort in surface quality 
optimization. In: F.-J. Villmer, \& E. Padoano (Eds.), Proceedings of the 7th International Conference Production Engineering and Management (pp. 111-113). Pordenone, Italy.

Elstermeyer, O. (2017). Process chain development for Rapid tooling. Master's Thesis, OWL University of Applied Sciences Lemgo, Università degli Studi di Trieste.

Flynn, J. M., Shokrani, A., Newman, S. T., \& Dhokian, V. (2016). Hybrid additive and subtractive machine tools-Research and industrial developments. International Journal of Machine Tools and Manufacturing, 101, 79-101.

Fousová, M., Kubásek, J., Dvorskỳ, D., \& Machová, M. (2015). 3D printing as an alternative to casting, forging and machining technologies? Manufacturing Technology, 15(5), 809-814.

Fraunhofer ILT. (2015). Reduction of surface roughness of slm components by means of modulated laser radiation, Annual Report Fraunhofer Institute for Laser Technology, 71.

Gibson, I., Rosen, D., \& Stucker, B. (2010). Additive manufacturing technologies: Rapid prototyping to direct digital manufacturing. New York: Springer.

Harrison Electropolishing L.P. Ra \& RMS Surface Roughness Calculation. Surface Finish Formulas. http://www.harrisonep.com/elect ropolishing-ra.html. Accessed 15 December 2016.

Karunakaran, K. P., Suryakumar, S., Pushpa, V., \& Akula, S. (2010). Low cost integration of additive and subtractive processes for hybrid layered manufacturing. Robotics and Computer-Integrated Manufacturing, 26, 490-499.

Karunakaran, K. P., Bernard, A., Suryakumar, S., Dembinski, L., \& Taillandier, G. (2012). Rapid manufacturing of metallic objects. Rapid Prototyping Journal, 18(4), 264-280.

Lauwers, B., Klocke, F., Klink, A., Tekkaya, A. E., Neugebauer, R., \& McIntosh, D. (2014). Hybrid processes in manufacturing. CIRP Annals Manufacturing Technolgy, 63(2), 561-583.

Masanta, M., Ganesh, P., Kaul, R., Nath, A. K., \& Choudhury, A. R. (2009). Development of a hard nano-structured multi-component ceramic coating by laser cladding. Materials Science and Engineering A, 508, 134-140.

Menges, G. (1991). Spritzgieß-Werkzeuge. Hanser: Anleitung zum Bau von Spritzgieß-Werkzeugen.

Mycroft, W., Katzman, M., Tammas-Williams, S., Hernandez-Nava, E., Panoutsos, G., Todd, I., \& Kadirkamanathan, V. (2020). A data-driven approach for predicting printability in metal additive manufacturing processes. Journal of Intelligent Manufacturing, $31,1769-1781$.

Nestler, K., Böttiger-Hiller, F., Adamitzki, W., Glowa, G., Zeidler, H., \& Schubert, A. (2016). Plasma electrolytic polishing-An overview of applied technologies and current challenges to extend the polishable material range. Procedia CIRP, 42, 503-507.

Newman, S. T., Zhu, Z., Dhokia, V., \& Shokrani, A. (2015). Process planning for additive and subtractive manufacturing technologies. CIRP Annals Manufacturing Technology, 64(1), 467-470.

Newnes, L. (2015). Experimental framework for testing the finishing of additive parts.
Ning, J., Sievers, D. E., Garmestani, H., \& Liang, S. Y. (2019). Analytical modeling of in-process temperature in powder bed additive manufacturing considering laser power absorption, latent heat, scanning strategy, and powder packing. Materials, 2019(12), 808.

Parfenov, E. V., Yerokhin, A., Nevyantseva, R. R., Gorbatkov, M. V., Liang, C.-J., \& Matthews, A. (2015). Towards smart electrolytic plasma technologies: An overview of methodological approaches to process modeling. Surface and Coatings Technology, 269, $2-22$.

Penchev, P., Bhaduri, D., Dimov, S., \& Soo, S. L. (2014). Novel manufacturing platform for scale up production of miniaturized components. In Proceedings of the 9th international workshop on microfactories (pp. 231-238).

Scheideler, E. M., Villmer, F.-J., Adam, G., Timmer, M. (2016) Topology optimization and additive manufacturing.-A perfect symbiosis? In: Villmer, F.-J., Padoano, E. (Eds.), Proceedings of the 6th international conference production engineering and management (pp. 27-38), Lemgo, Germany.

Tillmann, W., Schaak, C., Nellesen, J., Schaper, M., Aydinöz, M. E., \& Hoyer, K.-P. (2017). Hot isostatic pressing of IN718 components manufactured by selective laser melting. Additive Manufacturing, 13, 93-102.

Unterberg, P., Realizer GmbH, Germany. (2016). Experiences with SLM. Borchen, Bad Salzuflen, Germany.

Vandenbroucke, B., \& Kruth, J.-P. (2007). Selective laser melting of biocompatible metals for rapid manufacturing of medical parts. Rapid Prototyping Journal, 13(4), 196-203.

Verein Deutscher Ingenieure e.V. (2013). VDI-Richtlinie 3405 (part 2). Additive manufacturing processes, rapid manufacturing, Beam melting of metallic parts. Qualification, quality assurance and post processing 2013 Beuth, Berlin, Germany.

Wang, H. Y., Jiang, Q. C., Zhao, Y. Q., Zhao, F., Ma, B. X., \& Wang, Y. (2004). Fabrication of TiB2 and TiB2-TiC particulates reinforced magnesium matrix composites. Materials Science and Engineering $A$, 372, 109-114.

Yasa, E., \& Kruth, J. (2011). Application of laser re-melting on selective laser melting parts. Advances in Production Engineering \& Management, 4, 259-270.

Zhong, M., Liu, W., \& Zhang, H. (2006). Corrosion and wear resistance characteristics of $\mathrm{NiCr}$ coating by laser alloying with powder feeding on grey iron liner. Wear, 260, 1349-1355. https://doi. org/10.1016/j.wear.2005.09.033.

Zwan, R. (2012). Current trends in technology and society, vol 1 (pp. 46-58). Brisbane: Primrose Hall Publishing Group.

Publisher's Note Springer Nature remains neutral with regard to jurisdictional claims in published maps and institutional affiliations. 